\title{
Knowledge management: Role of the the Radiation Safety Information Computational Center (RSICC)
}

\author{
Timothy Valentine ${ }^{\mathrm{a}}$ \\ Radiation Safety Information Computational Center, Oak Ridge National Laboratory, One Bethel Valley Road, Oak Ridge, \\ Tennessee 37831, USA
}

\begin{abstract}
The Radiation Safety Information Computational Center (RSICC) at Oak Ridge National Laboratory (ORNL) is an information analysis center that collects, archives, evaluates, synthesizes and distributes information, data and codes that are used in various nuclear technology applications. RSICC retains more than 2,000 software packages that have been provided by code developers from various federal and international agencies. RSICC's customers (scientists, engineers, and students from around the world) obtain access to such computing codes (source and/or executable versions) and processed nuclear data files to promote on-going research, to ensure nuclear and radiological safety, and to advance nuclear technology. The role of such information analysis centers is critical for supporting and sustaining nuclear education and training programs both domestically and internationally, as the majority of RSICC's customers are students attending U.S. universities. Additionally, RSICC operates a secure CLOUD computing system to provide access to sensitive export-controlled modeling and simulation (M\&S) tools that support both domestic and international activities. This presentation will provide a general review of RSICC's activities, services, and systems that support knowledge management and education and training in the nuclear field.
\end{abstract}

\section{Introduction}

For the past five decades, the Radiation Safety Information Computational Center (RSICC) has served as the official repository for nuclear modeling and simulation $(\mathrm{M} \& \mathrm{~S})$ and data for the Department of Energy (DOE) and its predecessors and has collected and disseminated related information worldwide under specific distribution restrictions and guidelines set forth by the US government. RSICC maintains collaborations with other similar international organizations to foster cooperation and exchange of M\&S tools and data to benefit to our customers. RSICC houses nearly 2,000 software packages provided by code developers supported from various research institutes and universities in the US, as well as international agencies and research centers. Many of these codes have a broad range of applications and uses.

One revolutionary challenge that RSICC has faced is the ever-expanding capability of computing technology accompanied by growing reliance on the need for $M \& S$ tools. In some part, the demand and reliance on $M \& S$

\footnotetext{
a e-mail: valentinete@ornl.gov

This manuscript has been authored by UT-Battelle, LLC under Contract No. DE-AC05-00OR22725 with the U.S. Department of Energy. The United States Government retains and the publisher, by accepting the article for publication, acknowledges that the United States Government retains a non-exclusive, paid-up, irrevocable, world-wide license to publish or reproduce the published form of this manuscript, or allow others to do so, for United States Government purposes. The Department of Energy will provide public access to these results of federally sponsored research in accordance with the DOE Public Access Plan (http://energy.gov/downloads/doe-public-access-plan).
}

tools is a consequence of the increasing cost associated with operation of experimental nuclear facilities and the reduced availability of such facilities. Therefore, being able to provide quality-controlled software and data that can be utilized across a diverse set of computing technologies is of growing importance, yet it is no easy task. Fortunately, RSICC has had the support of sponsors and code and data developers, along with access to a variety of computing resources to ensure that the packages that we supply to the user community span the breadth of computing resources available to our customers and address the range of the applications for which such software is needed.

RSICC's distribution of M\&S tools and data helps to promote international cooperation in nuclear safety, ensures the safe development and deployment of nuclear technology, and provides those countries possessing or pursuing nuclear technology access to state-of-the-art software. All of these activities are an integral part of the knowledge management mission of RSICC. In fact, RSICC has over 16,000 active customers. Students, researchers, and faculty at U.S. universities represent the fastest growing segment of RSICC's customers. The growth in the number of RSICC's foreign customers has presented some challenges in providing access to M\&S tools that have deemed "sensitive" by the U.S. Federal government because these pose a greater risk of diversion from their intended purposes or could be utilized for nefarious activities. To help to resolve the dilemma between the open sharing of nuclear technology and the need to minimize the potential use of nuclear technology for nefarious purposes, RSICC developed, deployed, and implemented a secure computing system to provide access 


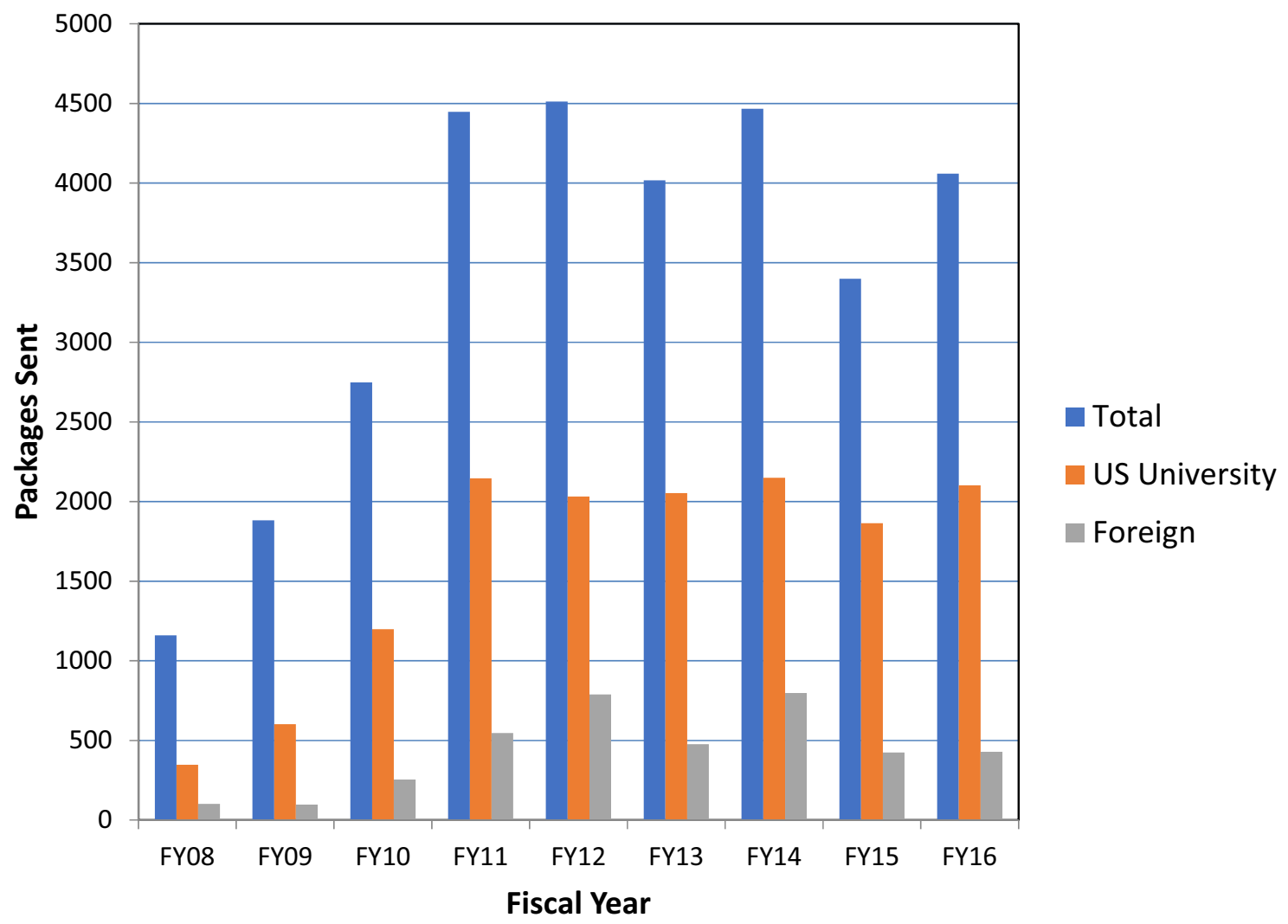

Figure 1. RSICC's annual software package distribution.

to modern software and data for which access would otherwise be limited or restricted. The deployment of this system also has additional benefit because some users may lack access to sufficient computing infrastructure to effectively utilize modern M\&S tools. This secure access provides an important avenue for knowledge sharing. This paper provides a general overview of RSICC's activities, services, and systems that support knowledge management activities in the nuclear field.

\section{Software demand}

The demand for state-of-the-art M\&S tools has nearly doubled since 2009, as shown in Fig. 1. Since fiscal year 2011, RSICC has distributed approximately 4,000 software and data packages annually to its customers with the exception being in fiscal year 2015. Data for fiscal year 2016 represents only three quarters of the fiscal year. RSICC has also seen a substantial growth in the number of packages delivered to customers who are not US citizens (shown in the figure as "foreign") as compared to fiscal years prior to 2010. During this same time, RSICC has seen substantial growth in the number of requests from US universities as compared to fiscal years prior to 2010 .

Along with the growth in the demand for $M \& S$ tools and data, RSICC's customer base has expanded to include over 100 countries and over 20,000 registered individuals. The greatest number of our customers is in the US, but RSICC has seen additional growth over the past 5 years in countries pursuing development and deployment of nuclear technology. Over 16,000 active customers from throughout the World have requested or received software from RSICC since 2005. Outside the
US, most of RSICC's clients reside in Canada, France, the United Kingdom, China, South Korea, and Germany. As reliance on advanced $M \& S$ tools and data grows, RSICC anticipates further growth in the demand for its services. The provision of these services is critical to the preservation and promulgation of knowledge in the nuclear field.

\section{Knowledge management of modeling and simulation tools and data}

RSICC's primary mission is centered on the archival, preservation and distribution of software, data and experimental databases. The $M \& S$ software and data distributed by RSICC are being used for many applications including but not limited to developing advanced reactor concepts, computing radiation source terms, designing and developing fusion devices, ensuring nuclear criticality safety, designing accelerators, nuclear medicine studies, and nuclear security applications. Of the 2,000 packages maintained by RSICC, the Los Alamos Monte Carlo code MCNP®[1] and the Oak Ridge National Laboratory SCALE system [2] are in the most demand by our customers, as shown in Fig. 2, because these codes can be applied across many application areas. Accordingly, RSICC also co-sponsors various training workshops and seminars to enhance the knowledge of our customers in the use of these codes.

In addition to the M\&S software and data, RSICC also archives, maintains and distributes databases and information from legacy experiments related to nuclear criticality safety, reactor physics and nuclear shielding. These databases such as SINBAD [4] are maintained in 


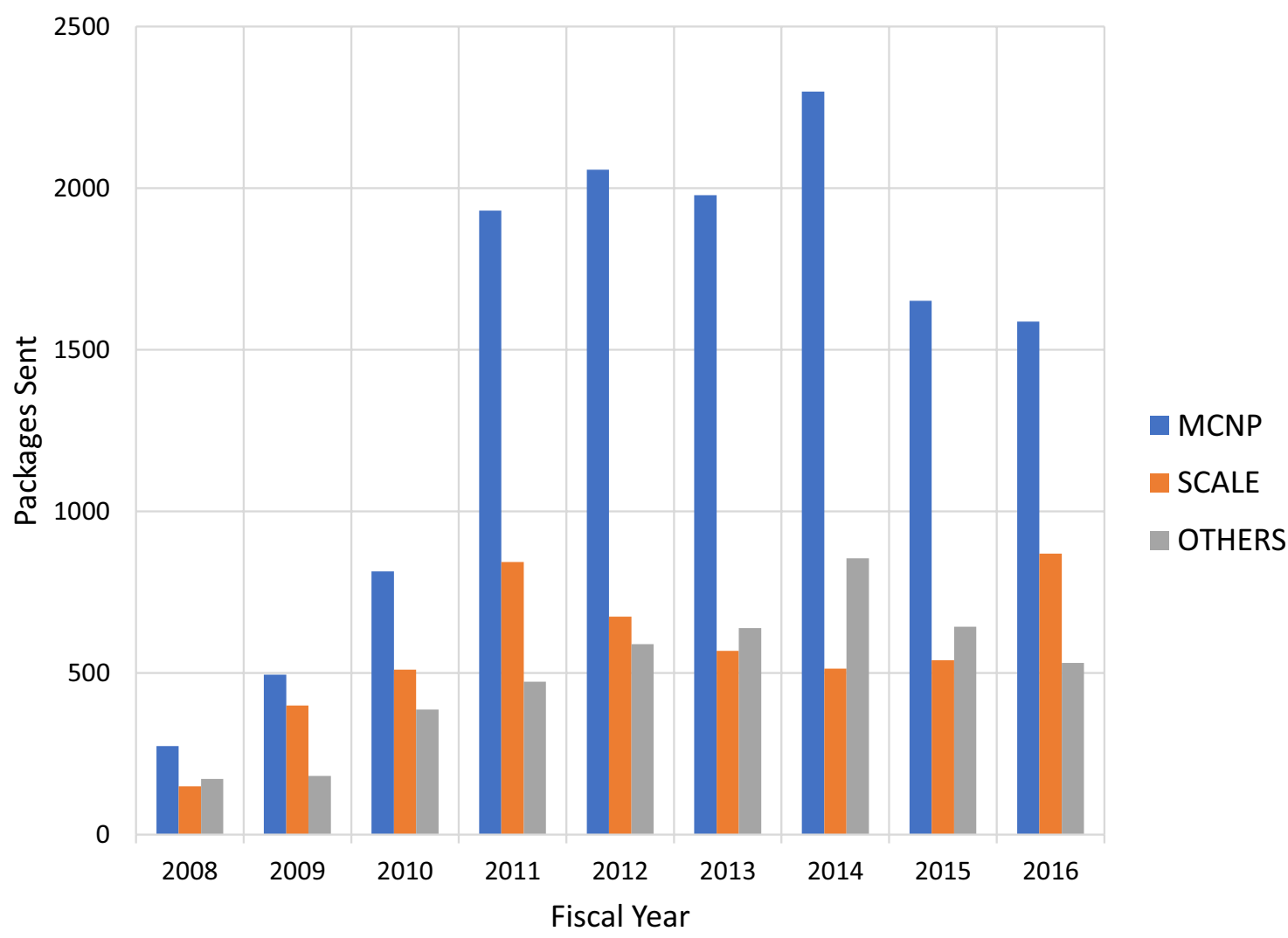

Figure 2. High-demand package distributions by RSICC.

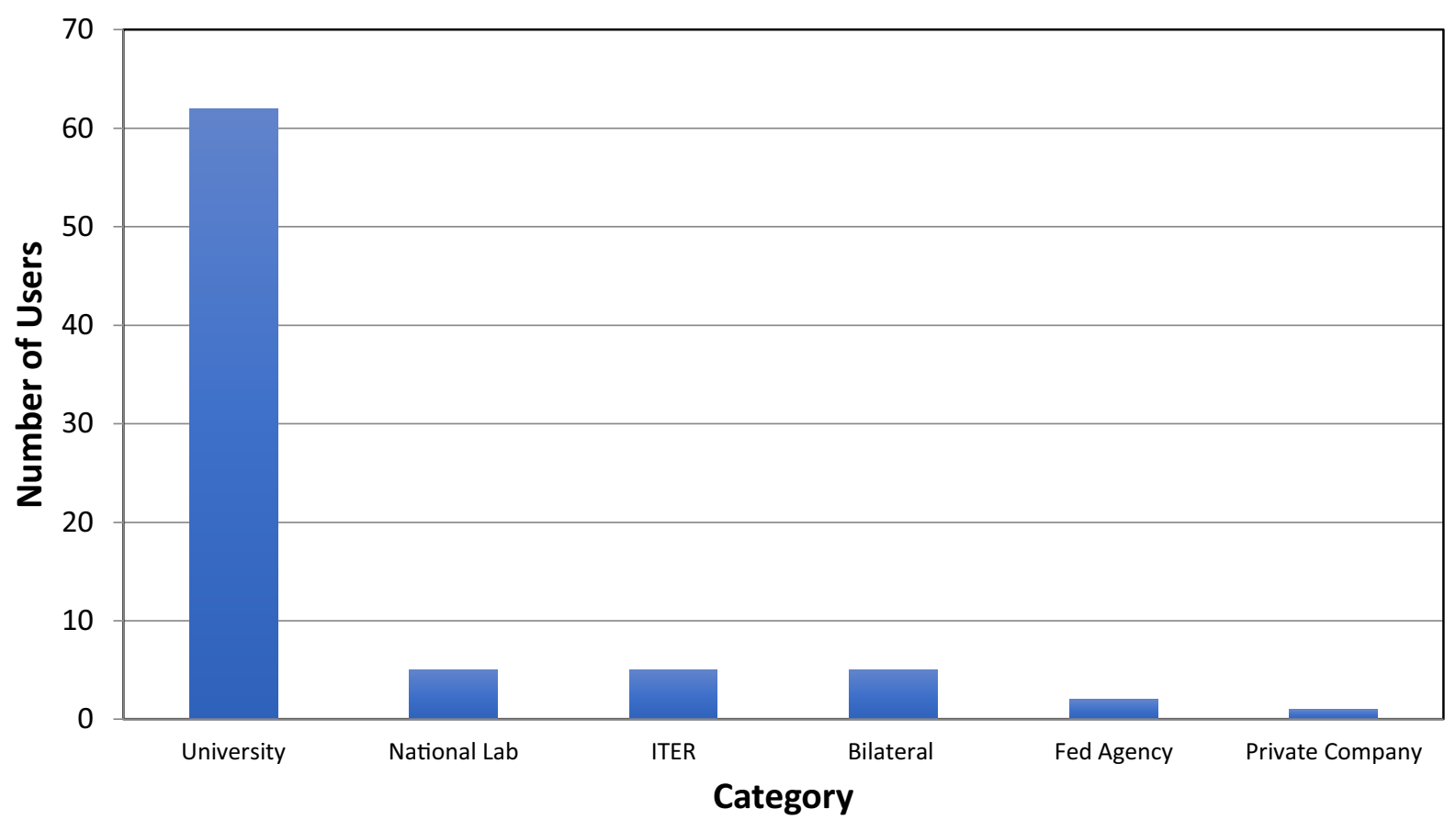

Figure 3. Users of RSICC's secure cloud server.

electronic format and provided to our customers to support on-going research and education activities. Some of these databases are jointly maintained by RSICC and the Data Bank of the Nuclear Energy Agency.

As previously stated, some individuals who otherwise could not be provided access to certain $M \& S$ software are now be able to use state-of-the art M\&S software through RSICC's secure cloud computing system. These individuals are mainly foreign nationals collaborating with or working at US national laboratories, universities, or companies. Access to RSICC's secure cloud server has been provided to over 80 individuals involved in various research and development activities. The majority of the individuals that are accessing the secure system are foreign 
nationals attending U.S. universities as shown in Fig. 3. Another example is the international collaboration under ITER [3], the international nuclear fusion megaproject under construction in the south of France. Access to the secure cloud has been provided to allow ITER contributors to use the $\mathrm{MCNP}^{\circledR}$ Monte Carlo code for the design of shielding and safety systems without which it would be difficult for them to meet their obligations to this international project.

\section{Summary}

Knowledge management is an integral part of RISCC's core mission. RSICC's acquisition, preservation and dissemination of M\&S tools, data and legacy experimental data along with the operation of secure cloud server contribute significantly to the promulgation of knowledge in the use of state-of-the-art M\&S tools.

Additionally, RSICC's support of conferences, training workshops, and seminars that are related to $M \& S$ tools is vital to enhancing the knowledge base of the users of M\&S tools. Centers such as RSICC play a pivotal role in knowledge management and are a valuable service to nuclear community. In light of the growing dependency on $\mathrm{M} \& S$, such centers are likely to have an even greater role in knowledge management in the future.
The author would like to acknowledge the support of the US Department of Energy Office of Nuclear Energy, the National Nuclear Security Administration, and the Office of Nonproliferation and Arms Control for development and deployment of the secure cloud computing system. The author would like to thank Mark Baird, Matt Disney, Ken Barker, and Brian Zachary of ORNL for their efforts to install, test, and validate the system's operation, as well as for their development of the network security protocols that enabled the system to be deployed.

\section{References}

[1] J.T. Goorley, et al., "Initial MCNP6 Release Overview," Nucl. Tech. 180, 298 (2012)

[2] SCALE: A Comprehensive Modeling and Simulation Suite for Nuclear Safety Analysis and Design, ORNL/TM-2005/39, Version 6.1, Oak Ridge National Laboratory, Oak Ridge, Tennessee (2011)

[3] B.J. Greene, "ITER: burning plasma physics experiment," Plasma Phys. Control Fusion 45, 687 (2003)

[4] I. Kodeli, et al., "SINBAD - Shielding Benchmark Experiments - Status and Planned Activiites," Proc. ANS $14^{\text {th }}$ Biennial Topical Meeting of Radiation Protection and Shielding, Carlsbad, New Mexico (April 3-6, 2006) 